\title{
ESTUDO ANATÔMICO DO LENHO \\ DE COLLIGUAYA BRASILIENSIS KLOTZSCH EX BAILL. (EUPHORBIACEAE) ${ }^{1}$
}

\author{
ANELISE MARTA SIEGLOCH ${ }^{2}$ SIDINEI RODRIGUES DOS SANTOS ${ }^{3}$ \\ JOSÉ NEWTON CARDOSO MARCHIORI ${ }^{4}$
}

\section{RESUMO}

O lenho de Colliguaya brasiliensis Klotzsch ex Baill. (Euphorbiaceae) é presentemente descrito, com vistas a contribuir para o conhecimento estrutural das espécies reófilas. O estudo foi realizado a partir de uma amostra de madeira coletada em Santa Maria, Rio Grande do Sul. Foram observados: poros solitários e em múltipos radiais, muito pequenos e muito numerosos; elementos vasculares curtos, com placas de perfuração simples, pontoações intervasculares alternas e espessamentos espiralados; parênquima axial seriado, em arranjos apotraqueal difuso e paratraqueal escasso; raios unisseriados, predominantemente heterogêneos; e fibras não septadas, com pontoações simples e paredes muito finas. Apesar de relativamente comuns na família, não foram observados canais intercelulares e cristais na madeira. No tocante à anatomia de reófitas, a espécie investigada reúne todas as características atribuídas a estas plantas, com exceção de fibras gelatinosas. Palavras-chaves: Colliguaya brasiliensis, Euphorbiaceae, anatomia da madeira, reofilia.

\section{ABSTRACT \\ [Wood anatomy of Colliguaya brasiliensis Klotzsch ex Baill. (Euphorbiaceae)].}

The wood anatomy of Colliguaya brasiliensis Klotzsch ex Baill. (Euphorbiaceae) is described, with the aim to contribute to the structural knowledge on rheophile plants. The study was developed with wood samples collected in the municipality of Santa Maria, Rio Grande do Sul state - Brasil. The following features were observed: solitary and radial multiple pores, very small and numerous; short vessel elements, with simple perforation plates, alternate intervessel pits and spiral thickenings; strand axial parenchyma, in apotrachealdiffuse and paratracheal-scanty arrangements; uniseriate heterogeneous rays; and non-septate fibers, with simple pits and very-thin walls. Intercellular channels and crystals were not observed, despite their common occurrence in the botanical family. According to the rheophilic status, the wood structure showed several features that are usually attributed to these kind of plants, with the exception of gelatinous fibers.

Key words: Colliguaya brasiliensis, Euphorbiaceae, wood anatomy, rheophilic plants.

\section{INTRODUÇÃO}

Bastante numerosa e heterogênea, a família Euphorbiaceae reúne cerca de 300 gêneros e 7300 espécies de árvores, arbustos, ervas e lianas, geralmente latescentes (Marchiori, 2000). No Brasil, são aproximadamente 70

\footnotetext{
1 Recebido para publicação em 05-12-2010 e aceito para publicação em 06-01-2011.

2 Acadêmica do Curso de Graduação em Engenharia Florestal, bolsista de iniciação científica do PIBIC-CNPQ, Universidade Federal de Santa Maria. CEP 97105900. Santa Maria, RS, Brasil. anesiegloch@yahoo.com.br

3 Biólogo, bolsista do CNPq - Brasil, doutorando do Programa de Pós-Graduação em Engenharia Florestal, Departamento de Ciências Florestais, Universidade Federal de Santa Maria, RS, Brasil.

4 Engenheiro Florestal, Dr. Bolsista de Produtividade em Pesquisa $(\mathrm{CNPq}$ - Brasil). Professor Titular do Departamento de Ciências Florestais, Universidade Federal de Santa Maria. Santa Maria, RS, Brasil.
}

gêneros e 1.000 espécies, o que torna a família uma das mais numerosas e complexas da flora nativa, sob o ponto de vista taxonômico (Souza e Lorenzi, 2005).

Colliguaya brasiliensis Klotzsch ex Baill. integra o contingente dos "sarandis", espécies que habitam a beira de rios e apresentam adaptações para suportar a correnteza das águas (Marchiori, 2000). Trata-se de árvore pequena ou arbusto de até $5 \mathrm{~m}$ de altura e $20 \mathrm{~cm}$ de DAP, com tronco flexível e copa umbeliforme, pequena e paucifoliada. As folhas, linearlanceoladas $(5-10 \mathrm{~cm} \times 6-10 \mathrm{~mm})$ e de bordo serrilhado, ao contrário da maioria dos representantes da família, não apresentam glândulas, látex ou tricomas. Única espécie do gênero no sul do Brasil, Colliguaya brasiliensis 
ocorre naturalmente na Depressão Central do Rio Grande do Sul e, menos comumente, no noroeste e nordeste do Planalto (Alvarez, 1977; Marchiori, 2000).

As espécies reófilas são muito importantes para a proteção de nascentes e barrancas, além de servirem como abrigo e fonte de alimento para a ictiofauna. Mais recentemente, com o desenvolvimento da Bioengenharia, tais plantas passaram a ser vistas como alternativa para a estabilização e proteção de taludes fluviais, com bons resultados (Durlo \& Sutile, 2005).

O conhecimento da estrutura anatômica das reófilas é indispensável para a compreensão de seu comportamento, quando submetidas à força das águas. A composição do lenho, bem como a estrutura e organização de seus elementos constituintes, reflete-se diretamente em propriedades físicas da madeira (Esau, 1959), caso da flexibilidade, uma das características mais desejadas na proteção de taludes fluviais (Vollsinger et al., 2000).

Apesar de sua importância, são notavelmente escassas as informações sobre espécies reófilas, sobretudo do ponto de vista estrutural. A descrição anatômica de Colliguaya brasiliensis, neste sentido, visa a preencher uma lacuna no conhecimento científico, além de contribuir para a maior difusão da espécie em trabalhos de Bioengenharia.

\section{REVISÃO DE LITERATURA}

A anatomia das Euphorbiaceae apresenta grande variabilidade devido à diversidade de hábitos vegetais e ambientes ocupados por suas numerosas espécies (Mecalfe \& Chalk, 1972).

Record \& Hess (1949) relacionam, para a família: poros em múltiplos, com tendência a alinhamento radial; vasos com placas de perfuração simples, pontoações intervasculares alternas e sem espessamentos espiralados; raios heterogêneos, finos e inconspícuos, usualmente unisseriados; parênquima axial reticulado ou em faixas concêntricas; fibras geralmente muito longas, com pontoações simples ou aréolas reduzidas, de paredes finas até muito espessas, comumente gelatinosas e raramente septadas; canais radiais presentes em muitos gêneros; e cristais frequentemente presentes em raios, no parênquima axial ou em ambos.

Para as Crotonoideae, subfamília a qual pertence o gênero em estudo, constam os seguintes caracteres anatômicos: poros solitários e em múltiplos radiais; elementos vasculares moderadamente curtos a médios, com placas de perfuração simples e pontoações intervasculares médias a grandes; parênquima axial abundante, apotraqueal, com poucas células isoladas ou formando estreitas faixas contínuas (1-2 células), por vezes em associação com parênquima paratraqueal; séries parenquimáticas de até 4 , mais comumente 8 células; raios exclusivamente unisseriados ou com 2-3 (5) células de largura; raios usualmente com menos de $1 \mathrm{~mm}$ de altura, embora menores em muitos gêneros; raios heterogêneos, frequentemente com mais de 10 fileiras marginais de células eretas e mais de uma parte multisseriada; fibras com pontoações usualmente simples, por vezes com pequenas aréolas, de comprimento médio a moderadamente curtas e paredes finas a espessas; e laticíferos e canais intercelulares por vezes presentes, em raios (Metcalfe \& Chalk, 1972).

Para o gênero Colliguaya, não foram encontradas referências anatômicas. No caso de outros gêneros ou espécies nativas de Euphorbiaceae, recomendam-se: Barros \& Callado (1997), Barros et al. (2001; 2003), Marchiori (2000) e Marchiori et al. (2009; 2010).

Sobre a anatomia das reófilas, como já salientado, são escassas as referências na literatura. Denardi (2007), em um dos poucos trabalhos sobre o tema, relaciona, com base no estudo de Phyllanthus sellowianus, Sebastiania schottiana, Salix humboldtiana e Salix x rubens, uma série de características estruturais associadas à "síndrome anatômica de reofilia": vasos pequenos $(<100 \mu \mathrm{m})$, solitários ou em múltiplos radiais, com paredes de 2,5-5,0 $\mu \mathrm{m}$ de espessura; parênquima axial pouco abundante ou ausente; raios estreitos; elementos vasculares e fibras de 400 e $700 \mu \mathrm{m}$ de comprimento, 
respectivamente; fibras gelatinosas, presentes; e tecido lenhoso constituído por $10-30 \%$ de vasos, $0-5 \%$ de parênquima axial, $10-20 \%$ de raios e $60-70 \%$ de fibras.

\section{MATERIAL E MÉTODOS}

O material estudado foi coletado na localidade de Arroio Grande, interior do município de Santa Maria, Rio Grande do Sul. A amostra de madeira e respectivo material botânico foram incorporados à Xiloteca e Herbário do Departamento de Ciências Florestais (HDCF) da Universidade Federal de Santa Maria, sob o número 6087.

Do material lenhoso foram extraídos três corpos de prova $(3 \times 3 \times 3 \mathrm{~cm})$ da parte mais externa do lenho, próxima ao câmbio, orientados para obtenção de cortes nos planos transversal, longitudinal radial e longitudinal tangencial. Um outro bloco foi também retirado, com vistas à maceração.

No preparo das lâminas de cortes anatômicos seguiu-se a técnica padrão no Laboratório de Anatomia da Madeira da Universidade Federal do Paraná: as amostras foram amolecidas por fervura em água e seccionadas em micrótomo de deslizamento, regulado para a obtenção de cortes com espessura nominal de $20 \mu \mathrm{m}$. Estes foram tingidos com safra-blau, desidratados em série alcoólica-ascendente $(30 \%, 50 \%, 70 \%$, 95\% e duas vezes em álcool absoluto) e montados em lâminas permanentes, com "Entellan".

Para as lâminas de macerado, adotou-se o método de Franklin, modificado (Kraus \& Arduin, 1997) e coloração da pasta com safranina $1 \%$. A montagem de lâminas seguiu o anteriormente descrito, com a diferença de que as três primeiras etapas foram desenvolvidas sobre papel de filtro.

A descrição microscópica da madeira baseou-se nas recomendações do IAWA Committee (1989). No caso da percentagem dos tecidos, foram realizadas 600 determinações ao acaso, com auxílio de contador de laboratório, conforme proposto por Marchiori (1980). A frequiência de poros foi obtida de forma indireta, a partir de um quadrado de área conhecida superposto a fotomicrografias de seções transversais da madeira. As medições foram realizadas em microscópio Carl Zeiss, com ocular de escala graduada, no Laboratório de Anatomia da Madeira da Universidade Federal de Santa Maria. Nas características quantitativas, os números entre parênteses equivalem aos valores mínimos e máximos observados. $\mathrm{O}$ valor que acompanha a média é o desvio padrão. As fotomicrografias foram tomadas em microscópio Olympus cx 40 , equipado com câmera digital Olympus Camedia c3000, no laboratório de Anatomia da Madeira da Universidade Federal do Paraná, a quem os autores agradecem.

\section{DESCRIÇÃO ANATÔMICA}

Anéis de crescimento: indistintos ou fracamente delimitados por fina camada de fibras radialmente estreitas. Porosidade difusa (Figura 1A,B).

Vasos: muito numerosos a extremamente numerosos $\left(84 \pm 30(50-119)\right.$ poros $\left./ \mathrm{mm}^{2}\right)$, ocupando $8 \pm 2,4 \%$ do volume da madeira. Conteúdos, ausentes. Poros solitários e em múltiplos radiais 2 - 7 (12), de seção angular/ poligonal, muito pequenos $(31,2 \pm 7,0(20-$ $42,5) \mu \mathrm{m})$ e de paredes finas $(2,4 \pm 0,35(1,9-$ $3,1) \mu \mathrm{m}$ ) (Figura 1A,B). Elementos vasculares curtos $(340 \pm 83(150-480) \mu \mathrm{m})$, com placas de perfuração simples, oblíquas ou verticais, e apêndices em uma ou em ambas as extremidades. Espessamentos espiralados conspícuos na parede. Pontoações intervasculares alternas, pequenas $(6,4 \pm 0,7(5,1-7,2)$ $\mu \mathrm{m})$, circulares ou ovais, com abertura em fenda inclusa, não ornamentada (Figura 1D). Pontoações raio-vasculares semelhantes, em tamanho e forma, às intervasculares $(6,2 \pm 0,7$ $(5,1-7,2) \mu \mathrm{m})$, mas com bordas reduzidas ou aparentemente simples (Figura 1D).

Parênquima axial: representando $5 \pm 0,9 \%$ do volume da madeira; pouco distinto em relação as fibras e em arranjo apotraqueal difuso, 

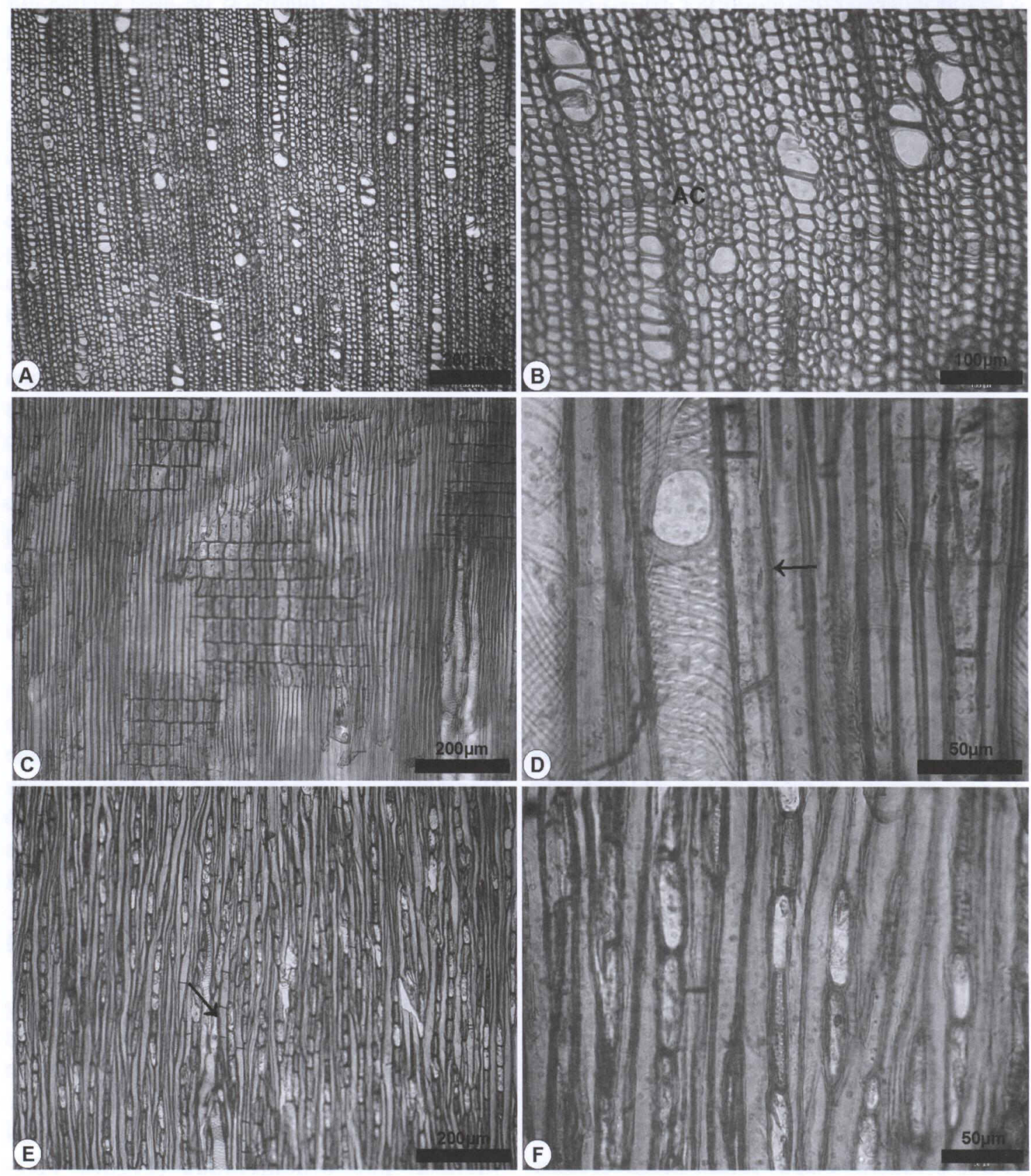

FIGURA 1 - Fotomicrografias da madeira de Colliguaya brasiliensis. A - Plano transversal, mostrando porosidade difusa e poros solitários e em múltiplos radiais. B - Mesma seção, com limite de anel de crescimento pouco distinto (AC) e fibras de paredes finas. C - Raios heterogêneos, de células eretas e quadradas, em seção longitudinal radial. D - Detalhe de vaso com espessamentos espiralados, placa de perfuração simples e parênquima paratraqueal (seta), em seção longitudinal radial. E-Aspecto geral da seção longitudinal tangencial, destacando série parenquimática com 4 células (seta). F - Mesma seção, em maior aumento, mostrando raios exclusivamente unisseriados. 
raro difuso-em-agregados, além de paratraqueal escasso (Figura 1A,B,D). Séries parenquimáticas com (2) $3-4$ (5) células e $372 \pm 58$ (275 - 502) $\mu \mathrm{m}$ de altura (Figura 1E).

Raios: muito numerosos $(19 \pm 3(14-24)$ raios $/ \mathrm{mm}$ ), ocupando $23 \pm 2,5 \%$ do volume da madeira; compostos de células exclusivamente eretas ou, mais comumente, por uma mistura de células eretas e quadradas, raro procumbentes (Figura 1C). Raios exclusivamente unisseriados, de $269 \pm 129(150-670) \mu \mathrm{m}$ e (1) 5 - 8 (12) células de altura (Figura 1E,F). Células radiais de paredes disjuntas, presentes. Conteúdos, ausentes.

Fibras: representando $64 \pm 3,2 \%$ do volume da madeira. Fibras com pontoações simples e aberturas cruzadas, frequentemente exclusas, visíveis nas faces radiais e tangenciais da parede; são curtas $(724,8 \pm 87(590-950) \mu \mathrm{m})$, com 22,5 $\pm 2,3(17,5-28,7) \mu \mathrm{m}$ de largura e de paredes muito finas $(3,5 \pm 0,60(2,5-5) \mu \mathrm{m})$ (Figura 1B). Fibras septadas, fibras gelatinosas, espessamentos espiralados e traqueídeos, ausentes.

Demais caracteres: variantes cambiais, tubos laticíferos e taníferos, canais intercelulares, máculas, células oleíferas ou mucilaginosas, estratificação e cristais, ausentes.

\section{ANÁLISE DA ESTRUTURA ANATÔMICA}

As principais características anatômicas do lenho de Colliguaya brasiliensis estão de acordo com as possibilidades estruturais relacionadas por Record \& Hess (1949) e Metcalfe e Chalk (1972) para as Euphorbiaceae: poros solitários e em múltiplos radiais, muito pequenos e muito numerosos; elementos vasculares curtos, com placas de perfuração simples e pontoações intervasculares alternas; parênquima axial seriado, em arranjo apotraqueal difuso e paratraqueal escasso; raios unisseriados, predominantemente heterogêneos; e fibras não septadas, com pontoações simples e paredes muito finas.

Presentes na madeira em estudo, os espessamentos espirados não são referidos por Record \& Hess (1949) para a família. Metcalfe
\& Chalk (1972), todavia, mencionam a ocorrência deste caráter para certas espécies da subfamília Crotonoideae, à qual pertence a espécie em estudo. Cabe lembrar, todavia, que nenhum material de Colliguaya foi examinado pelos referidos autores.

Apesar de relativamente comuns na família, não foram observados canais intercelulares e cristais na madeira investigada, bem como fibras gelatinosas. Nas Euphorbiaceae, segundo Record \& Hess (1949) e Metcalfe \& Chalk (1972), a presença destes detalhes anatômicos é típica para madeiras com fibras de paredes espessas, motivo pelo qual se justifica a ausência do caráter em Colliguaya brasiliensis, espécie com fibras de paredes muito finas.

No tocante à reofilia, cabe destacar, de início, que a flexibilidade é a característica mais importante no caso de plantas submetidas à força constante da correnteza. Diversos estudos demonstram que espécies com menor desenvolvimento de tronco e ramos são geralmente mais flexíveis do que as que apresentam caules espessos; ramos jovens, do mesmo modo, são mais flexíveis do que ramos velhos, cujas paredes celulares são mais lignificadas.

Weitzer et al. (1998), em testes de flexão estática do caule de algumas espécies lenhosas usadas em Bioengenharia, observaram que, independentemente da espécie, quanto maior o diâmetro, menor é o valor dos módulos de elasticidade e ruptura. Denardi (2007), por sua vez, em estudo anatômico e da flexibilidade do caule de quatro espécies lenhosas, comprovou que, independentemente da espécie, a flexibilidade dos caules diminui com o aumento do diâmetro, o que implica, com o passar do tempo, em relativa perda de proteção para os taludes fluviais. A manutenção de plantas jovens (flexíveis) nas margens de cursos de água, deste modo, assume fundamental importância.

Do ponto de vista anatômico, a presença e/ ou abundância de certos tecidos ou células influencia diretamente nas propriedades mecânicas da madeira (Burger \& Richter, 1991). 
No caso da flexibilidade, são especialmente importantes a presença e abundância de tecidos parenquimáticos (pouco ou não lignificados). Fibras de paredes finas, da mesma forma, tornam a estrutura mais flexível, devido à menor quantidade de parede celular por unidade de volume. Em pesquisa com Salix viminalis (Salicaceae) e algumas espécies do gênero Calamus (Arecaceae), Garay (2002) observou que o caule da primeira é o menos flexível, atribuindo a diferença ao maior peso específico.

$\mathrm{Na}$ questão da flexibilidade, a ocorrência ou não de fibras gelatinosas assume grande importância. Este tipo especial de fibras, rica em celulose e com pouca lignina, é comum na estrutura anatômica de lianas (Vieira, 1994) e outras dicotiledôneas lenhosas com tronco ou ramos inclinados, bem como no caule de plantas sujeitas a grandes esforços de sustentação (Apezzato-da-Glória \& Carmello-Guerreiro, 2004) e à força da água (Marchiori, 2004); integrante do chamado lenho de reação (tração, neste caso), as fibras gelatinosas conferem maior flexibilidade à madeira, impedindo o rompimento de tecidos.

Apesar do escasso parênquima axial, a madeira em estudo apresenta alta percentagem de raios e fibras de paredes finas, aspectos que contribuem para a maior flexibilidade da madeira; não foram observadas, todavia, fibras gelatinosas. Cabe salientar que o lenho de tração não se caracteriza apenas pela presença da camada G, mas, principalmente, pela ausência de lignificação na parede das fibras. Este critério, aliás, é tido como mais preciso do que a simples observação de fibras gelatinosas, pois certas madeiras, embora desprovidas do caráter, exibem propriedades de lenho de tração. Se este é (ou não) o caso de Colliguaya brasiliensis, não se pode afirmar no presente estudo. Além destes aspectos, a ocorrência de troncos relativamente finos em Colliguaya brasiliensis, contribui, igualmente, para a maior flexibilidade do caule.

A "síndrome anatômica de reofilia", proposta por Denardi (2007), resulta da combinação de diversos detalhes anatômicos que, em sua maioria, foram observados na madeira de Colliguaya brasiliensis. Confirma-se, portanto, no presente trabalho, a "síndrome anatômica de reofilia", proposta por Denardi (2007). Na espécie em estudo, todavia, não foram observadas fibras gelatinosas, aspecto salientado pelo autor como importante para a flexibilidade dos caules; a ausência do mesmo, como anteriormente discutido, pode dever-se tanto à distribuição do caráter na família, como à própria espessura da parede das fibras.

\section{REFERÊNCIAS BIBLIOGRÁFICAS}

ALVAREZ, A.F. Estudo das tribos Acalypheae Müll. Arg. e Hippomaneae Reichenb. (Euphorbiaceae) no Rio Grande do Sul - Brasil. Dissertação (Mestrado em Botânica) Universidade Federal do Rio Grande do Sul, Porto Alegre, 1977.

APPEZZATO-DA-GLÓRIA, B.; CARMELLOGUERREIRO, S.M. Anatomia vegetal. Viçosa: UFV, 2003. $438 \mathrm{p}$.

BARROS, C.F.; CALLADO, C.H. Madeiras da mata atlântica. Anatomia do lenho de espécies ocorrentes nos remanescentes florestais do estado do Rio de Janeiro, Brasil. Rio de Janeiro: Instituto de Pesquisas Jardim Botânico do Rio de Janeiro, 1997. 86 p.

BARROS, C.F.; CALLADO, C.H.; MARCON, M.L.; COSTA, C.G.; CUNHA, M.; LIMA, H.R.P.; MARQUETE, O. Madeiras da Mata Atlântica. Anatomia do lenho de espécies ocorrentes nos remanescentes florestais do estado do Rio de Janeiro, Brasil. Rio de Janeiro: Instituto de Pesquisas Jardim Botânico do Rio de Janeiro, 2001. 94 p.

BARROS, C.F.; CALLADO, C.H.; CUNHA, M.; MARCON, M.L.F.; TAMAIO, N.; MARQUETE, O.; COSTA, C.G. Madeiras da Mata Atlântica. Anatomia do lenho de espécies ocorrentes nos remanescentes florestais do estado do Rio de Janeiro, Brasil. Rio de Janeiro: Instituto de Pesquisas Jardim Botânico do Rio de Janeiro, 2003. 86 p.

BURGER, L.M.; RICHTER, H.G. Anatomia da Madeira. São Paulo: Nobel, 1991. 154 p. 
DENARDI, L. Anatomia e flexibilidade do caule de quatro espécies lenhosas para o manejo biotécnico de cursos de água. $112 \mathrm{f}$. Tese (Doutorado em Engenharia Florestal) Universidade Federal de Santa Maria. Santa Maria, 2007.

DURLO, M.A.; SUTILI, F.J. Bioengenharia: manejo biotécnico de cursos de água. Porto Alegre: EST, 2005. 189 p.

ESAU, E. Anatomia vegetal. Barcelona: Omega, 1959. $729 \mathrm{p}$.

GARAY, R. Caracterizacion fisico-mecánica de Salix spp. Silvicultura y Producción, Santiago, p. 222240, 2002.

IAWA COMMITTEE. IAWA list of microscopic features for hardwood identificacion. IAWA Bulletin, v. 10, n. 2, p. 218-359, 1989.

KRAUS, J.E.; ARDUIN, M. Manual básico de métodos em Morfologia Vegetal. Rio de Janeiro: EDUR, 1997. 198 p.

LAWRENCE, G.H.M. Taxonomia das plantas vasculares. Lisboa: Fundação Caulouste Gulbenkian, v. 2, p. 854, 1977.

MARCHIORI, J.N.C. Estudo anatômico do xilema secundário de algumas espécies dos gêneros Acacia e Mimosa, nativas no Estado do Rio Grande do Sul. 1980. 186 f. Dissertação (Mestrado em Engenharia Florestal) Universidade Federal do Paraná. Curitiba, 1980.

MARCHIORI, J.N.C. Dendrologia das Angiospermas: das Bixáceas às Rosáceas. Santa Maria: Editora da UFSM, 2000. 240 p.

MARCHIORI, J.N.C. Fitogeografia do Rio Grande do Sul: Campos Sulinos. Porto Alegre: EST, 2004. $110 \mathrm{p}$.

MARCHIORI, J.N.C; MUÑIZ, G.I.B; SANTOS,
S.R. Madeiras do Rio Grande do Sul: 1 Descrição microscópica de 33 espécies nativas. Santa Maria: [s.n.], 2009. 80 p.

MARCHIORI, J.N.C; MUÑIZ, G.I.B; SANTOS, S.R. Madeiras do Rio Grande do Sul: 2 Descrição microscópica de 35 espécies nativas. Santa Maria: Anaterra, 2010. 80 p.

METCALFE C. R.; CHALK L. Anatomy of the Dicotyledons. Oxford: Clarendon Press, 1972. $1500 \mathrm{p}$.

RECORD, S.J.; HESS, R.W. Timbers of the New World. New Haven: Yale University Press, 1949. $640 \mathrm{p}$.

SOUZA, V.C.; LORENZI, H. Botânica sistemática: guia ilustrado para identificação das famílias de Angiospermas Dicotiledôneas da flora brasileira, baseado em APG II. Nova Odessa: Instituto Plantarum, 2005. 640 p.

VIEIRA, R.C. Estrutura do caule de Bauhinia radiata Vell. em diferentes ambientes. Revista Brasileira de Biologia, v. 54, n. 2, p. 293-310, 1994.

VOLLSINGER, S.; DOPPLER, F.; FLORINETH, F. Ermittlung des stabilitatsverhaltens von Ufergehölzen in Zusammenhang mit Erosionsprozessen an Wildbächen. Wien: Bundesministerium für Land-und Forstwirtschaft, Umwelt und Wasserwirtschaft, 2000.107 p. WEITZER, C.H.; DOPPLER, F.; FLORINETH, F. Untersuchungen über die Wirksamkeit von Pflanzen in Einzugsgebieten des Forsttechnischen Dienstes der Wildbach- und Lawinenverbauung. Arbeistsbereich Ingenieurbiologie u. Landschaftsbau, Univ. f. Bodenkultur Wien, Hrsg. Bundesministerium $\mathrm{f}$. Land- u. Forstwirtchaft, Gruppe V/C, Wien, 1998. $81 \mathrm{p}$. 